\title{
Characterization of Tactical Departure Scheduling in the National Airspace System
}

\author{
Alan Capps ${ }^{1}$ \\ Mosaic ATM, Fort Worth, TX, 76155 \\ Shawn A. Engelland ${ }^{2}$ \\ NASA Ames Research Center, Fort Worth, TX 76155
}

\begin{abstract}
This paper discusses and analyzes current day utilization and performance of the tactical departure scheduling process in the National Airspace System (NAS) to understand the benefits in improving this process. The analysis used operational air traffic data from over 1,082,000 flights during the month of January, 2011. Specific metrics included the frequency of tactical departure scheduling, site specific variances in the technology's utilization, departure time prediction compliance used in the tactical scheduling process and the performance with which the current system can predict the airborne slot that aircraft are being scheduled into from the airport surface. Operational data analysis described in this paper indicates significant room for improvement exists in the current system primarily in the area of reduced departure time prediction uncertainty. Results indicate that a significant number of tactically scheduled aircraft did not meet their scheduled departure slot due to departure time uncertainty. In addition to missed slots, the operational data analysis identified increased controller workload associated with tactical departures which were subject to traffic management manual re-scheduling or controller swaps. An analysis of achievable levels of departure time prediction accuracy as obtained by a new integrated surface and tactical scheduling tool is provided to assess the benefit it may provide as a solution to the identified shortfalls. A list of NAS facilities which are likely to receive the greatest benefit from the integrated surface and tactical scheduling technology are provided.
\end{abstract}

\section{Introduction}

$\mathrm{N}$ ASA's current Integrated Arrival/Departure/Surface research portfolio includes integration of surface information with en route departure scheduling. The Precision Departure Release Capability (PDRC) activity is assessing the value of using surface trajectory-based takeoff (OFF) time predictions for departure scheduling. Companion papers ${ }^{1,2}$ present a concept overview and results from benefits assessment studies.

This paper describes the NAS shortfalls that PDRC technology seeks to address and assesses current PDRC levels of predictive accuracy against the current need. The document begins by describing a nation-wide survey of current tactical departure scheduling operations. Existing system shortfalls are then examined via a discussion of system performance along with the measurement approach and corresponding results. The shortfalls discussion is followed by a description of the current levels of OFF time prediction accuracy that can be obtained in the PDRC system today. The paper concludes with a discussion of sites most likely to benefit from PDRC technology.

\section{Current Day Tactical Departure Scheduling}

In order to identify existing shortfalls which may be eliminated with reduced departure prediction uncertainty, it is necessary to have an understanding of the current day tactical departure scheduling process. This section covers the following five topics: 1) Tactical departure scheduling overview, 2) Current Inbound Tactical Departure

\footnotetext{
${ }^{1}$ National Airspace System Engineer, NASA/FAA North Texas Research Station, AIAA Senior Member.

${ }^{2}$ Aerospace Engineer, NASA/FAA North Texas Research Station, AIAA Senior Member
} 
Scheduling Capability, 3) Current Outbound Tactical Departure Scheduling Capability, and 4) Tactical versus strategic departure scheduling.

\section{A. Tactical Departure Scheduling Overview}

Tactical departure scheduling is the process used by ATC to regulate air traffic flow to eliminate local demand/capacity imbalances and satisfy local traffic management initiatives (TMIs). Tactical departure scheduling is not required during normal NAS operations as the airspace into which the flight is being released generally has sufficient capacity to accommodate the departure. However, during periods of high demand or low capacity for the airspace being scheduled into, tactical departure scheduling may be utilized.

Tactical departure scheduling in the NAS today can be divided into two distinct tactical scheduling modes, which are outbound scheduling of departures from an airport within the departure Air Route Traffic Control Center (ARTCC, hereafter referred to as "Center") to a remote Center and inbound scheduling of departures into an arrival stream of a Traffic Management Advisor (TMA) metered airport. The inbound and outbound terms are generic labels for tactical departure scheduling functions provided by existing decision support tools (i.e. TMA scheduling, 'internal' scheduling, 'adjacent' scheduling, 'coupled' scheduling, extended metering, etc.) The flight length associated with the tactical timeframe varies somewhat in the literature. The authors chose an upper bound of 90 minutes as the guideline for flight lengths subject to tactical departure scheduling. This flight length was chosen in part based upon information obtained from operational data usage of the decision support tools that support tactical departure scheduling.

Figure 1 illustrates the relationship of the Dallas/Fort Worth (DFW) departure airport relative to arrival metering to Houston Intercontinental (IAH) airport. Given that DFW resides within the IAH metering freeze horizon and the limited airspace available to maneuver after departure prior to the outer meter arc, a high level of departure prediction accuracy is required. Later sections provide an estimate as to the level of predictive accuracy that is required.

Call For Release (CFR) is a common tactical departure scheduling procedure which requires Air Traffic Control Tower (ATCT) personnel to call the Center Traffic Management Unit (TMU) for a scheduled departure time prior to

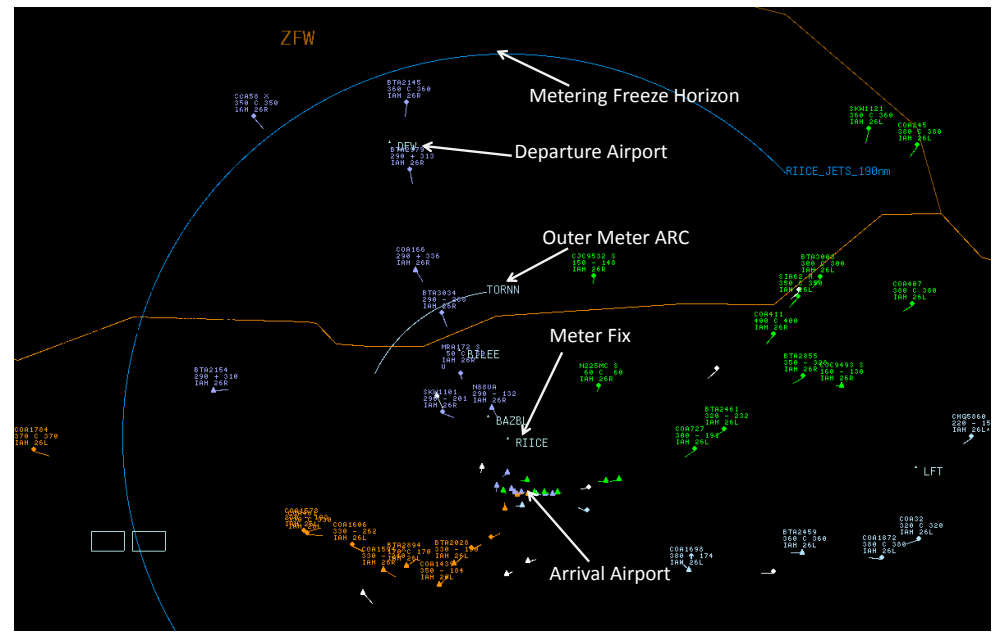

Figure 1. Inbound tactical scheduling geometry which requires a high level of departure prediction accuracy. releasing the aircraft for departure. The CFR procedure is applied to departing aircraft in order to ensure the demand placed on local airspace resources do not exceed the available capacity. In a CFR scenario it may or may not be necessary to delay the aircraft based upon the latest information available on the constrained flow at the time that an aircraft is ready to depart. The improved departure time compliance associated with the CFR procedure provides more accurate schedule predictions than are available via the aircraft's filed flight plan departure time (also known as Predicted Departure Time or PTIME) or by use of Expect Departure Clearance Times (EDCTs). EDCT times are generated by Traffic Flow Management (TFM) as a part of the strategic departure scheduling system and are not intended for tactical use. Aircraft PTIMEs represent a starting point from which the departure planning process begins but are historically prone to OFF time uncertainty. ${ }^{3,4}$ 
The required departure compliance window for CFR aircraft varies somewhat by facility. Today, no nationwide guidance exists, but based upon information obtained from traffic managers, generally inter-facility agreements call for flights to depart within a three minute window. This three-minute window is generally structured to allow departure two minutes prior to, or one minute later than, the target coordinated departure time. The idea of allowing the aircraft to depart two minutes early is that it is easier to delay the aircraft to fit into the constrained flow than to accelerate the aircraft to meet its scheduled time. Figure 2 provides an illustration of nationwide departure time compliance comparison between estimation methods available to TMCs during the month of January 2011. January was selected for operational data analysis primarily due to the availability and completeness of the TMA operational data set during this time period.

The values reported in Fig. 2 are the average absolute difference between the expected departure time and the actual departure time. The operational TMA data analyzed had information on aircraft PTIME, EDCT times, TMA times and actual departure times which were used for this nationwide departure time compliance analysis. An obvious difference exists in the departure time compliance between PTIME estimates, EDCT controlled times and CFR controlled times with the departure times coming from the CFR process providing the best compliance of the three. Using the CFR process during the month of

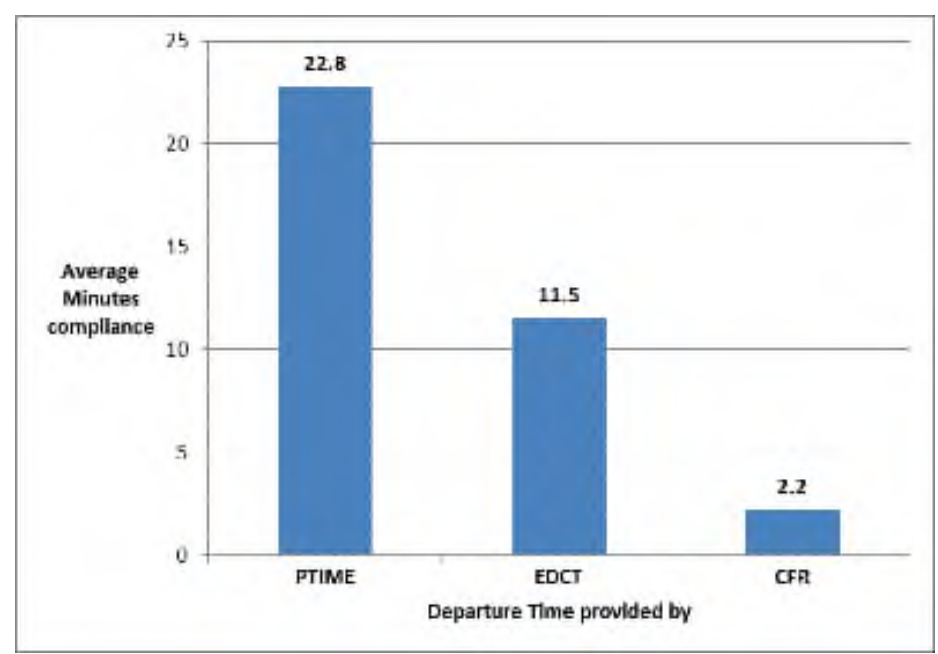

Figure 2. Average Nationwide Departure time compliance for January 2011.

January, approximately $69.2 \%$ of aircraft subject to CFRs in which TMA automation was utilized met the required $2 /+1$ window. In contrast, if EDCT times were required to meet a $-2 /+1$ window the compliance would have been approximately $20.4 \%$. Using PTIME compliance this percentage would drop to only $4 \%$ of flights that met the $2 /+1$ window.

\section{B. Inbound Tactical Departure Scheduling Capability}

As adjacent center metering has expanded the reach of TMA, the greatest need for departure scheduling capability has been for airports residing in another Center. Analysis of January 2011 operational data shows that

Table 1. Departure Scheduling from remote ARTCC Jan 2011.

\begin{tabular}{|l|c|c|}
\hline From Center & Into Center & $\begin{array}{l}\text { Number of Aircraft } \\
\text { Scheduled into } \\
\text { remote Center }\end{array}$ \\
\hline Jacksonville & Atlanta & 6267 \\
\hline Washington & Atlanta & 6072 \\
\hline Boston & New York & 3955 \\
\hline Washington & New York & 3719 \\
\hline Indianapolis & Atlanta & 3081 \\
\hline Cleveland & New York & 3012 \\
\hline Oakland & Atlanta & 2951 \\
\hline Los Angeles & Albuquerque & 2243 \\
\hline Memphis & Atlanta & 1619 \\
\hline Canada & New York & 1234 \\
\hline Indianapolis & New York & 469 \\
\hline Cleveland & Atlanta & 389 \\
\hline Albuquerque & Los Angeles & 384 \\
\hline Fort Worth & Houston & 382 \\
\hline Chicago & Cleveland & 210 \\
\hline Kansas City & Chicaco & 102 \\
\hline
\end{tabular}

$69.3 \%$ of all departure scheduling is performed from an origination Center that is different than the destination Center being scheduled into. Table 1 gives examples and frequency of usage of inbound tactically scheduled aircraft across Center boundaries during the month of January 2011. The "Number of Aircraft Scheduled into remote Center" lists the number of times a TMC from a Center other than the destination Center scheduled aircraft using TMA capability. Note that not all scheduling performed is from an adjacent center, for instance Indianapolis Center schedules into New York Center although the two Centers do not share a boundary. Another unique case occurs when aircraft departing Canadian airspace Call For Release into New York Center airspace.

The expanded scope of TMA usage is a factor to consider in analysis of tactical departure scheduling shortfalls, another factor is the effect that tactical departure scheduling capability has on the balance of delay that is assigned to the airborne stream versus airport surface. In December of 2005 a feature was added to TMA that allowed the TMC to determine whether or not departures should compete directly with active airborne flights. Prior to this feature, TMA always scheduled aircraft into the overhead stream in a manner that the departure had the same priority as airborne aircraft. The intent of this feature was to prevent airborne delays from reaching the point which it made it difficult for controllers to achieve the TMA meter crossing times. However, 
the tradeoff associated with limiting the airborne delays is an increase in departure delays. When the TMC chooses to delay the airborne flow, the TMA system will treat the departing aircraft with equal priority as airborne aircraft and assign a delay to unfrozen aircraft in the metered airborne stream if needed. In this situation, TMA may delay both the airborne stream and assign a ground delay to the departing aircraft. Analysis of the current usage based upon data from January 2011 indicates that the large majority (92\%) of flights scheduled in TMA took all of their tactical departure delay on the surface.

The ability for the TMC to determine whether the aircraft tactical delay should be taken airborne, on the surface, or a combination of the two is complicated by uncertainty in the scheduling process. Analysis of tactical departures scheduled into the arrival TMA system during metering indicates that approximately $21 \%$ of all scheduled aircraft experience both a TMA assigned ground delay and TMA assigned airborne delay. To prevent aircraft that are assigned delay on the airport surface from being delayed again once they join the airborne flow, the TMC may "freeze" the aircraft into the airborne flow when scheduling in TMA. If the TMC selects this option when scheduling a tactical departure, the TMA system will freeze the aircraft's scheduled time of arrival to the meter point thereby preventing any additional delay from being added to the aircraft once it becomes airborne. This feature allows the TMC to ensure the aircraft does not receive unplanned airborne delay; however, if the aircraft does not depart when expected and cannot achieve the time which is frozen into the arrival metering system's schedule, then the space that was being reserved for this aircraft will go unutilized barring additional action by ATC to prevent this from occurring. Currently, 29\% of departing flights that are scheduled into an arrival TMA system are scheduled frozen into the airborne flow: the remaining $71 \%$ of aircraft are allowed to adjust their position in the TMA arrival schedule upon first surveillance.

An additional shortfall of the current day inbound tactical departure scheduling system occurs when the tactical departure delays become very large. This situation may require Air Traffic Control System Command Center (ATCSCC) involvement. In the large majority of cases the assigned ground and airborne delay are small (i.e. less than 5 minutes $73 \%$ of the time in TMA), however, cases do exist in which airborne and/or ground delay is in excess of one hour. In the month of January there were approximately 20 occurrences of TMA assigned ground delays in excess of one hour. The majority of the examples of large TMA assigned ground delay were to either New York Center or Atlanta Center metered airports. In many cases, flights with high TMA-assigned surface delay also received an airborne delay from the TMA system. These examples of high ground delay with airborne delay may lend insight into why into why sites like New York Center and Atlanta Center are top users of the "schedule frozen" option previously discussed.

When high tactically-assigned ground delay occurs in the NAS, the ATCSCC may choose to implement an Airspace Flow Program (AFP) to regulate the flow of aircraft into the destination airport with the objective of reducing the TMA-assigned surface delays. The AFP scheduling scenario used for this purpose is unique in that it is designed to work in conjunction with the arrival TMA system; hence it is called a TMA Flow Program (TFP). The objectives of a TFP are to pre-condition the arrival stream such that TMA can utilize available space in the stream for tactical departure scheduling purposes. The boundaries of the TFP are set to be roughly contiguous with the arrival metering system's freeze horizon and any airport with departures inside of this boundary are exempt from the

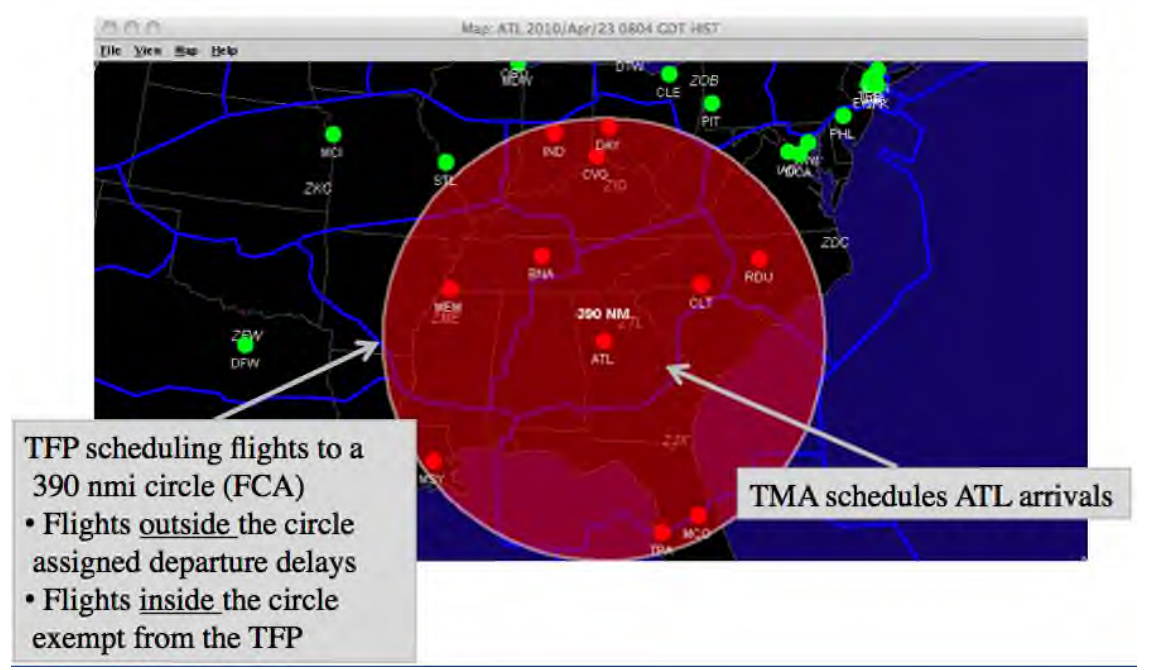
program. Using a TFP the TFM suite of tools assigns a ground delay to aircraft bound for the metered airport which are located outside of the red circle shown in Fig. 3, while TMA assigns a tactical ground delay (and potentially airborne delay depending on TMC selection) for those aircraft bound to the metered airport located within the red circle. ${ }^{5}$

Figure 3. Example of TMA Flow Program into Atlanta. 


\section{Outbound Tactical Departure Scheduling Capability}

In addition to the TMA arrival metering system, the Enroute Departure Capability (EDC) is now part of the tactical departure scheduling decision support tools available to TMC personnel. The EDC system design re-uses a number of common components of the arrival TMA system like its adaptation data structure, route processing algorithms and trajectory generation functions. While many of the core components of TMA have been leveraged to provide EDC capability, there are notable differences between arrival TMA and the EDC system.

The EDC system serves a different traffic management objective than the arrival TMA system. EDC's focus is outbound tactical departures leaving from one of the airports within a Center which are destined to a remote Center facility. In contrast the tactical departure scheduling capability in arrival TMA system is only focused on aircraft that are scheduled into its metered airports. EDC is commonly used to assist in the application of miles in trail restrictions between facilities, especially when the airspace being scheduled into is highly constrained or has multiple miles in trail initiatives to satisfy. An additional use of EDC is to assist in regulating departures into sectors which are experiencing high demand. In contrast, arrival TMA use is primarily motivated by the traffic volume in the arrival streams entering the metered airport rather than sector loading considerations.

The TMA EDC system is deployed to all 20 Centers within the NAS. Similar to the nationwide deployment of the arrival TMA system, there is significant variability in how EDC is used from one Center to another. As indicated by the blue portion of the bar chart in Fig. 4, the Center with the most frequent EDC usage is Boston Center, followed by Atlanta Center and Indianapolis Center. The combined usage of these three sites alone is greater than total EDC usage at all other Centers. Although Atlanta Center is the second largest user of EDC, the frequency of Atlanta's EDC usage is significantly less than that of inbound tactical departure scheduling into Atlanta's arrival TMA system. Figure 4 illustrates inbound and outbound tactical departure scheduling usage.

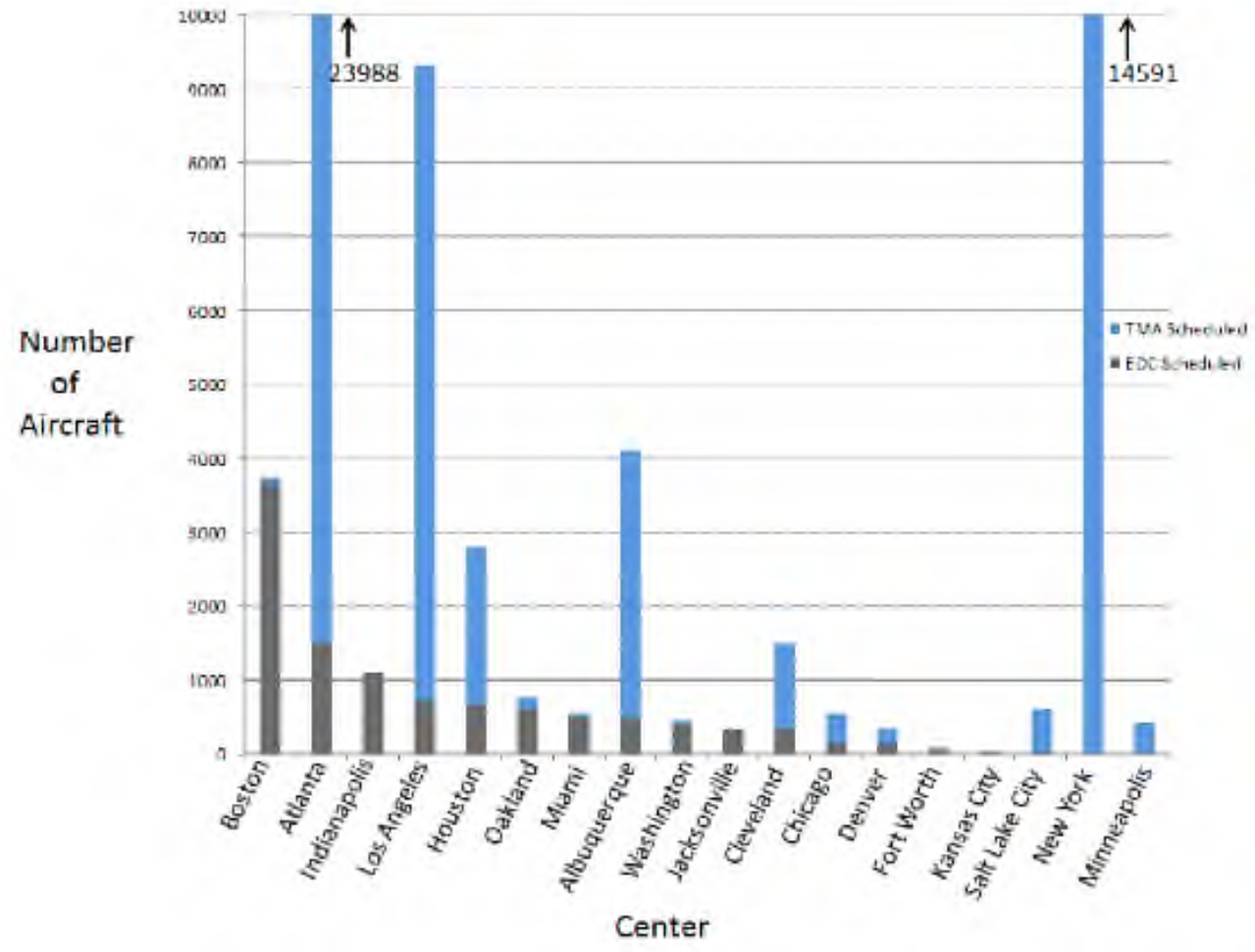

Figure 4. Tactical Scheduling of Arrival TMA and EDC - Jan 2011.

The total departure delays assigned by Arrival TMA versus EDC follow a similar model with inbound tactical departure scheduling assigning a total of 3,563 hours of surface delay to aircraft in the month of January 2011 versus a total of 480 hours of surface delay assigned by the outbound tactical departure scheduling system $(13.5 \%$ of inbound). 


\section{Tactical Versus Strategic Departure Scheduling}

While a significant amount of literature exists on the strategic departure scheduling process within the NAS which utilizes the Traffic Flow Management (TFM) suite of tools, information on the tactical departure scheduling process is quite limited. The two scheduling processes are distinct from one another and are currently not directly integrated. The strategic and tactical schedules have similar, but different objectives and usage characteristics.

A significant difference between tactical and strategic departure scheduling is the scope of the initiative. Strategic departure scheduling is focused on correcting large demand/capacity imbalances that exist in the NAS usually due to convective weather or high demand. This often requires significant delays over an extended period of time which may be assigned hours in advance of the affected aircraft's departure time. In contrast, tactical departure scheduling focuses on a specific air traffic flow that is subject to a local traffic management initiative (like Miles in Trail or Adjacent Center Metering) and generally introduces small delays to specific aircraft on an as-needed basis.

Tactical departure scheduling system delays are approximately 4 minutes per aircraft on average with a median of 1 minute, which is significantly lower than TFM delays with approximately 66 minute average and 52 minute median delays. These statistics are derived from January 2011 operational data. The difference in average delays is likely due to the national scope of TFM which must assign departure delay well in advance of departure, in contrast with tactical departure scheduling which applies delay on an as-needed basis to a single aircraft at a time. Tactical

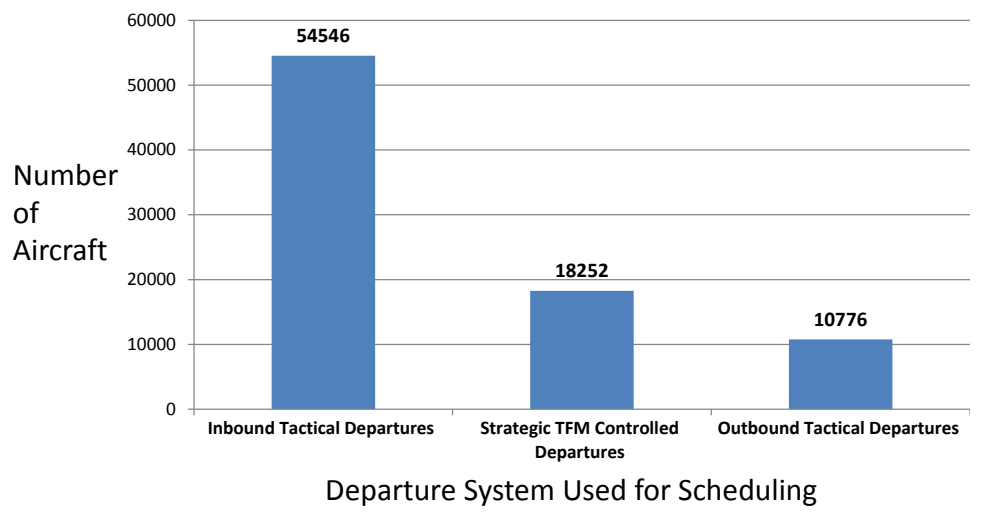

Figure 5. Departures Scheduled with Decision Support Tool - Jan 2011. departure schedules are able to consider the latest airspace conditions minutes before takeoff.

The frequency of use of tactical departure scheduling versus strategic as measured by the number of aircraft affected for January 2011 also varies significantly as illustrated in Fig. 5. The combined number of departures scheduled using the TMA and EDC tactical decision support tools (labeled "inbound" and "outbound" tactical departures in Fig. 5) was approximately $350 \%$ greater than aircraft affected by EDCTs (strategic TFM controlled departures). It is worth noting that inbound tactical departure scheduling (i.e. using arrival TMA) occurred significantly more frequently than outbound tactical departure scheduling (i.e. using EDC).

For this analysis, an aircraft was counted as being tactically scheduled only if the aircraft was both scheduled and 'accepted' or 'frozen' into the TMA Arrival or EDC system. A significant number of aircraft (approximately 18,489 during January, 2011) were initially scheduled in the TMA system but the scheduling process was not finalized by "accepting" or "freezing."

\section{NAS-wide Tactical Departure Scheduling Performance Analysis}

In addition to analyzing the January operational data, operational observations of scheduling performance were evaluated at DFW during the month of July 2011. Data from operational observations were used as a point of reference with which to test the data analysis measurement methodologies that were applied NAS-wide. This section discusses the metrics used for tactical departure scheduling performance and the results obtained in this analysis. Potential benefits due to reduced departure time uncertainty from PDRC can be quantified by the improvement in meeting a slot, reduction of manual intervention to mitigate missed or unattainable slots, and increased flight efficiency due to a reduction in airborne vectoring and speed controls.

\section{A. 'Hit Slot' Metric}

A key performance measurement in the tactical departure scheduling process is the efficiency with which available airspace in the constrained flow are being utilized by scheduled departure aircraft. Gaining insight into this measurement is important because it allows an objective means to analyze the utilization of tactical departure 
scheduling into the constrained overhead stream that may be lost due to departure prediction uncertainty. To obtain an assessment of slot utilization, operational data from the TMA and EDC systems were analyzed. A 'hit slot' measurement was created for this analysis. The objective of the 'hit slot' measurement is to determine whether or not the tactically scheduled departure joined the constrained flow at the sequence in which it was scheduled into prior to departure. This measurement allows an estimation of the effectiveness of the scheduling process based upon detailed scheduling information available in the operational TMA data. This section discusses details on the estimation approach used for this metric as well as results. Figure 6 provides an illustration of the 'hit slot'

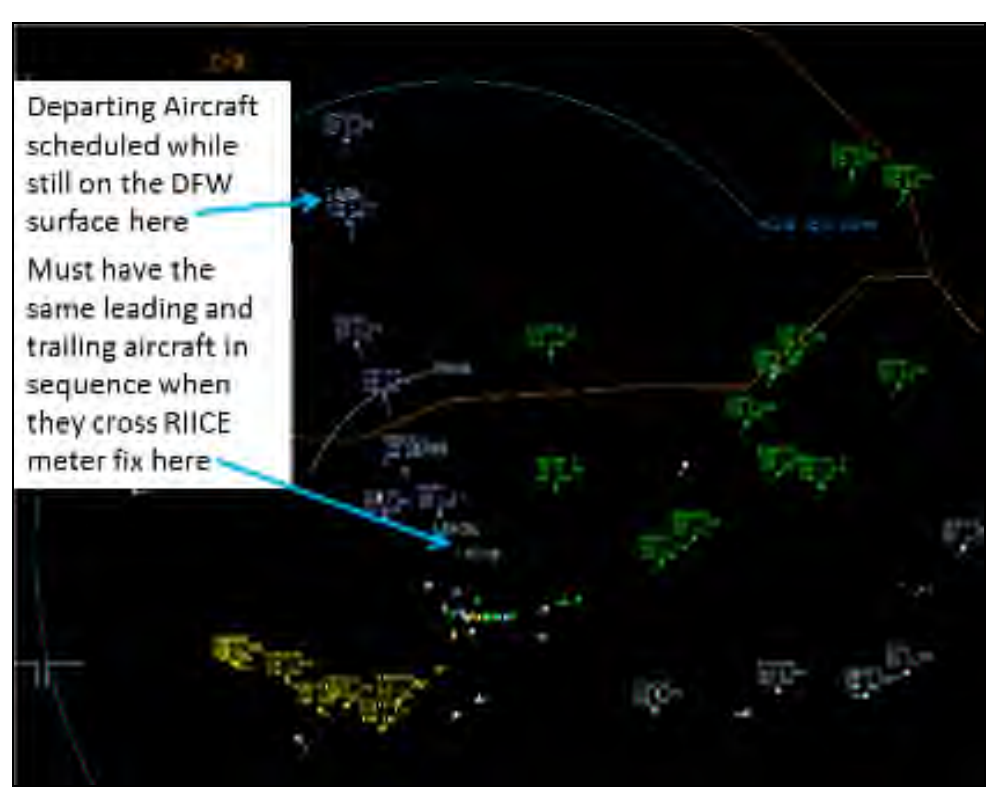

Figure 6. "Hit Slot" metric geometry for DFW to IAH Scheduling. measurement geometry for DFW to IAH tactical departure scheduling.

For the 'hit slot' measurement, the leading and trailing aircraft identification, TMA and EDC estimated times of arrival to the meter point (known as Meter point ETAs) and scheduled times of arrival to the meter point (known as Meter point STAs) were collected at the time at which the aircraft was scheduled in the operational TMA and EDC systems. Aircraft sequence and scheduling information were also collected at the point at which the aircraft received its first surveillance hit, and then again when it crossed the meter point location. The leading and trailing aircraft identification were examined to determine if they matched at each point in the aircraft's flight history from scheduling, to first track, to the actual sequence at crossing. An aircraft was said to hit its scheduled slot if its sequence relative to its leading and trailing aircraft remained when it was scheduled and when it crossed the meter point location. The same 'hit slot' sequencing analysis was repeated for each aircraft at the point at which surveillance was first acquired. This analysis measured whether or not the sequence provided by TMA and EDC after processing the first track hit matched the sequence at the actual meter fix crossing. This step was added to allow comparison of the difference in predictive accuracy between pre-departure scheduling versus attaining first surveillance.

An important consideration of the 'hit slot' measurement is determining the inclusion/exclusion criteria for aircraft to be used in the analysis. Aircraft which were excluded from the analysis included: 1) Aircraft which did not cross the meter point they were scheduled to due to lack of receipt of a crossing message, 2) Aircraft which did not have a record of leading and trailing aircraft at the point of scheduling, first track hit and crossing of the meter point based upon information available to the system at the point in time these events occurred 3) International tactical scheduling from Canada to NAS facilities given lack of departure time information available to TMA 4) Atlanta inbound tactical departure aircraft given the 'hybrid metering' scenario that Atlanta uses does not allow display of metered sequence, 5) Aircraft for which a Host departure message was not received 6) For arrival TMA only metered aircraft were included, 7) Only aircraft which the TMC scheduled and 'accept' or 'froze' were used.

To determine the sequence of aircraft at the times of interest mentioned above, the native stream class identification used by TMA and EDC was leveraged. For example, all jets scheduled over meter fix RIICE are a part of the RIICE_JETS TMA stream class. This information is made available in the native TMA data utilized for this analysis, as was the scheduled time of arrival to the meter fix (or meter point for EDC) for each stream class. The logic developed to support the 'hit slot' measurement ordered all aircraft by STA from lowest to highest, by stream class. This ordering was of all aircraft which were "scheduled" in the operational TMA or EDC system, which included any tactical departure schedules that had been scheduled at that time. Upon each schedule update the leading and trailing aircraft of every flight was identified assuming one existed. If an aircraft did not have a leading or trailing aircraft in the scheduler, these values were subsequently ignored in the analysis as previously mentioned. Upon occurrences of events of interest the sequence was stored along with the other aircraft metadata for later analysis. 
The results from the 'hit slot' analysis were separated into inbound (arrival TMA) versus outbound (EDC) tactical departure scheduling. A number of the results are represented as percentages due to inclusion/exclusion rules and data integrity checks. While certain aircraft had to be excluded to ensure data quality and that the measurements were on the right set of aircraft, the percentages are expected to hold true for the entire population of tactically scheduled departures in January due to the large sample size used for this analysis (over 22,400 aircraft after applying inclusion/exclusion logic).

Table 2 shows a high-level summary of the results from running the 'hit slot' measurement on all operational TMA and EDC facilities for the month of January. The "Hit Scheduled Slot \%" column represents the percentage of all tactically scheduled aircraft in January 2011 that had the same leading and trailing aircraft sequence when

Table 2. 'Hit Slot' measurement results for all operational TMA/EDC facilities during January 2011.

\begin{tabular}{|c|c|c|c|c|}
\hline System & $\begin{array}{c}\text { Hit Scheduled } \\
\text { Slot \% }\end{array}$ & $\begin{array}{c}\text { Hit First } \\
\text { Surveillance Slot \% }\end{array}$ & \% Difference & $\begin{array}{c}\text { Estimated Number of Aircraft that missed their slot } \\
\text { due to departure time prediction uncertainty }\end{array}$ \\
\hline Arrival TMA & 26.9 & 39.3 & 12.5 & 6792 \\
\hline EDC & 39.4 & 57.1 & 17.7 & 1911 \\
\hline
\end{tabular}

scheduled on the surface as when they crossed the meter point being scheduled to. The "Hit First Surveillance Slot $\%$ " provides this information but uses updated sequence obtained from TMA or EDC after the first surveillance is made available. The "\% Difference" takes the difference between the two hit slot percentages and then applies that percentage to all aircraft that were

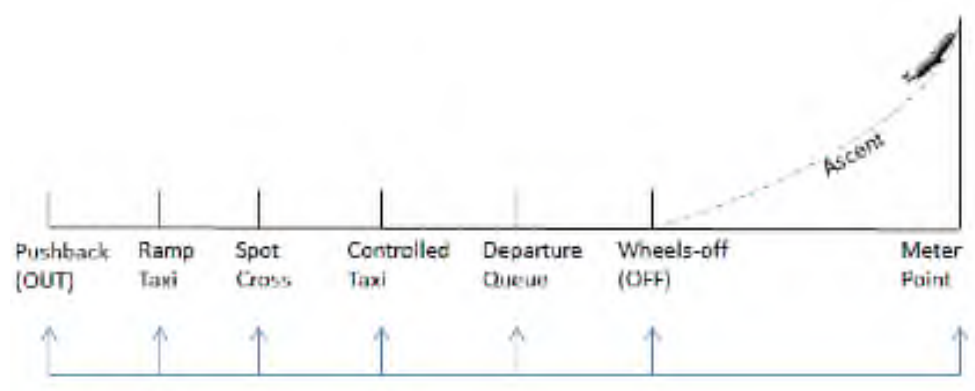

Figure 7. Tactical departure scheduling to the meter point incorporates cumulative uncertainty from a number of departure events. estimate to the TMA and EDC predictions immediately after wheels OFF when surveillance is first acquired. By capturing the estimates at these two time periods and comparing their difference, the ascent model portion of the prediction which is common between the two estimates, is isolated from the measurement.

While a goal of tactically scheduling an aircraft into a constrained flow is to identify and utilize resources ('slots') before the aircraft departs, the impact to the NAS which occurs when a scheduled slot is not met can vary. Observed cases of missed tactically scheduled departure slots indicate that they can often lead directly to lost capacity, most notably delay caused by the case in which an aircraft is scheduled frozen into an arrival TMA slot but does not meet its expected departure time window. Other observed impacts of missing the departure slot are inefficient flight paths due to required vectoring and/or speed controls (which can lead to excess fuel utilization) as well as increased controller and TMC workload (discussed in later section). According to the hit slot metric data obtained, approximately 1 in 4 aircraft hit their arrival slot in TMA, while more than 1 in 3 hit their slot in the EDC system. The primary reason for the difference is believed to be the size of the slot being scheduled into given that the average stream class separation difference in EDC is much larger than that of TMA. Based upon operational data from January 2011, the average stream class separation for arrival TMA is $8.2 \mathrm{~nm}$, while the average stream class separation in EDC is $23.6 \mathrm{~nm}$. The larger separation in EDC is consistent with intuition given that EDC's purpose is primarily to ensure MIT separations are met and the required separation being enforced is often quite large. The size of the slot being scheduled into is also believed to be the primary difference in percentage of aircraft that hit their scheduled slot in arrival TMA and EDC after the first track hit. As table 2 indicates there is a significant difference with EDC approximately 18\% more aircraft hitting the slot at this point in time versus arrival TMA. The percentage

8

American Institute of Aeronautics and Astronautics 
of aircraft that hit their slot after surveillance suggest that there may be room for improvement in the predictive capabilities of the ascent modeling of TMA and EDC. Future analysis may be warranted to analyze predictive accuracy of the ascent modeling due to aircraft weight, wind error, inaccurate routing, etc.

While, on average, aircraft hit their TMA-scheduled slots approximately $26.9 \%$ of the time, a fairly significant variation exists by site. The results of the hit slot metric were calculated for all TMA and EDC locations nationwide. The highest site percentage of the 'hit slot' measurement of all the arrival TMA systems was $32.9 \%$, while the lowest was $18.5 \%$ The highest site percentage of all EDC systems was $52.5 \%$, with the lowest being $22.7 \%$. The site specific variance may warrant additional consideration to determine the primary factors which lead to the variance. Given that the 'hit slot' percentage differs on a site by site basis, this suggests that the impact to the NAS may vary by facility as well.

\section{B. Arrival Metering Workload metric}

In addition to missed slots from departure time uncertainty, another shortfall to consider in current day tactical departure scheduling is the workload for the TMC and controllers. During the month of January 2011 approximately 153,426 flights had metering information delivered to sector controllers with the expectation that the controller would delay aircraft as necessary to meet the metered times. Of the metered aircraft, approximately 34,360 (22.4\%) were scheduled into the arrival stream using arrival TMA arrival scheduling capability. This represents a statistically significant portion of the overall metered aircraft during January.

The large sample of metered flights was analyzed to determine if manual intervention by either the sector controller or TMC during metering was higher for tactically scheduled departures than for flights which were not tactically scheduled. Three measures were utilized for this evaluation, which were the frequency controller swaps, controller resequences and individual aircraft reschedules by the TMC. The following gives a brief explanation of what these measures capture.

Sector controller tools associated with metering include two capabilities to control the sequence that TMA associates with arrival aircraft. These capabilities are known as swap and re-sequence. The swap capability allows the controller to identify any two aircraft on their display and exchange their meter point crossing times. This capability is used when the sector controller may disagree with the sequence or times that are being presented to him/her by the TMA system.

The tactically scheduled departure aircraft and the flights which were not tactically scheduled were analyzed to determine the frequency of required manual activity. The increased percentage of aircraft that required manual controller or TMC activity during metering suggests that tactical departure scheduling is a factor in increased workload for both sector controllers and TMCs. The highest increase of manual activity observed was the percentage increase of aircraft that undergo a single aircraft re-schedule. This measure showed a $6.1 \%$ increase for

Table 3. Percentage of aircraft which required manual intervention- Jan 2011.

\begin{tabular}{|l|c|c|c|c|}
\hline & & & & $\begin{array}{l}\text { Approximate \# Aircraft } \\
\text { subject to increased } \\
\text { manual activity }\end{array}$ \\
\hline Workload Category & Not Tactical Departure \% & Tactical Scheduled Departure \% & \% Difference \\
\hline Controller Swaps & 4.4 & 6.6 & 2.3 & 792 \\
\hline Controller Re-sequences & 4.4 & 6.0 & 1.7 & 572 \\
\hline Single Aircraft Re-schedule & 5.0 & 11.1 & 6.1 & 2125 \\
\hline
\end{tabular}

tactically scheduled departures over those aircraft which were not tactically scheduled. $\quad$ A summary of these results can be seen in Table 3.

\section{Effect of not scheduling a tactical departure into a constrained flow}

Observations of tactical scheduling performance from DFW into IAH during June and July of 2011 indicate that the benefit of increased departure time prediction accuracy may not be limited to the set of tactically scheduled departures previously discussed. Examples of these potential benefits were observed during PDRC engineering shadow evaluations. A typical example of this was for aircraft departing DFW with a destination of IAH which were not scheduled in the TMA system. In these examples the departing aircraft was sequenced ahead of several other aircraft in the stream class that were in close proximity. The addition of the departing aircraft added a 1 minute delay to the immediate trailing aircraft, which in turn added two minutes of delay to its trailing aircraft, and so on for a total of four aircraft which received airborne delay due to the departing aircraft. Vectoring off of nominal routes was visually observed in a number of these cases.

During PDRC observations in July, a number of occurrences were noted in which departures that were not tactically scheduled and coordinated between Center and ATCT personnel resulted in the use of speed controls and/or vectoring to accommodate the departing aircraft. During evaluations the "not scheduling" scenario which leads to this situation was discussed with Center personnel. Comments received indicate that while additional work 
is needed by sector controllers to accommodate uncoordinated departures, this is not viewed as an issue for sector controllers so long as other sector workload does not rise to a level of saturation that makes handling uncoordinated departure scheduling problematic. This information is consistent with previous research into the effect of "not scheduling" an aircraft into an arrival TMA flow. ${ }^{3,4}$ However, beyond the sector workload implications is the consideration of flight efficiency which effect fuel consumption. A coordinated departure release may have helped to reduce speed controls and vectoring which may in turn help reduce fuel consumption.

\section{Surface Departure Prediction Analysis}

The objective of PDRC is to leverage trajectory-based OFF time predictions to improve upon the current-day tactical departure scheduling process. Achieving this objective requires that one have accurate OFF time predictions from the surface system at the point in time which this information is required by the en route scheduling system. This section discusses a method to estimate the minimal required look-ahead time for OFF time predictions to satisfy tactical departure scheduling requirements. Also discussed are surface departure prediction accuracy requirements for present-day operations as well as recommendations for future surface analysis.

\section{A. Estimation of departure prediction look-ahead time requirement for Tactical Departure Scheduling}

In an ideal scenario, highly accurate aircraft wheels OFF times would be available to tactical and strategic planners hours ahead of the point at which the aircraft was ready to depart. In this ideal scenario all planners would be working from the same set of accurate information and making decisions that could be used to address local, regional, or national demand/capacity imbalances. However, highly accurate OFF times hours in advance of departure is not a feasible objective given the amount of pre-departure uncertainty which exists today., ${ }^{3,4,6,7}$ The cumulative effect of uncertainty from pushback prediction, through ramp taxi, spot transition, air movement area taxi, departure queue management, departure release, take off roll, ascent modeling, and forecast wind errors prior to reaching the meter crossing point provide a large amount of unpredictability. This uncertainty makes the departure planning process quite challenging.

While accurate wheels OFF estimates hours in advance may be an unrealistic objective in the NAS, providing accurate OFF time estimates minutes in advance of wheels OFF is an achievable objective which may help reduce or eliminate some of the challenges faced by tactical departure scheduling. An important question to consider for departure prediction accuracy is 'how far in advance of departure does the downstream scheduling system need to have accurate OFF time predictions?'

In order to estimate the minimal look-ahead time at which accurate OFF time predictions are required for aircraft departing into an arrival metering flow, one should consider the relative positions of the departure airport and the arrival metering freeze horizon. The geometry of the DFW-to-IAH metering scenario is illustrated in Fig. 8. DFW

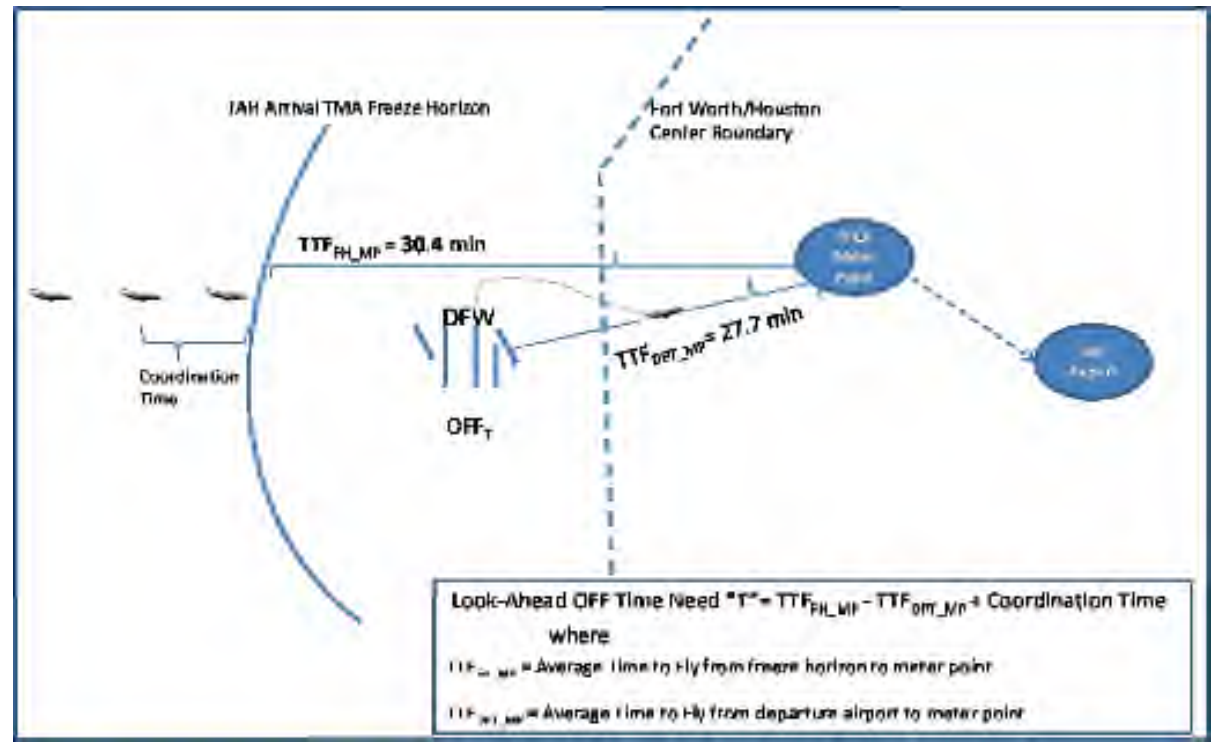

Figure 8. Method to estimate OFF prediction look ahead time need for DFW aircraft departing into Houston arrival metering. airport lies within the IAH arrival metering freeze horizon and the standard tactical departure scheduling procedure is to accept and freeze the aircraft into the arrival IAH flow to prevent the aircraft from receiving both a ground delay and an airborne delay. Due to this scheduling methodology, any surface or airborne prediction error in tactical departure scheduling to IAH during metering directly impacts the airborne arrival stream. For present-day operations this OFF time prediction is entirely manual. For the 
DFW to IAH metering scenario, the typical airborne aircraft scheduled into IAH over meter fix RIICE freezes at approximately 30.4 minutes prior to meter fix crossing when IAH traffic is in East flow, which is the predominant configuration used during metering at IAH. The typical flight time from DFW airport to the RIICE meter fix crossing is approximately 27.7 minutes. This means that an aircraft on the DFW surface which is ready to depart will be competing for slots with airborne aircraft whose schedules have been frozen on average for $30.4-27.7=2.7$ minutes (162 seconds). If the DFW aircraft are to compete with unfrozen aircraft for a slot into the constrained flow then the tactical scheduling process must occur at least 162 seconds prior to departure. The 162 second figure represents a theoretical minimum for the tactical departure scheduling lead time. Additional time is required for the Center TMU to consider the schedule and communicate the release time to ATCT. Some time is also required for the TMA scheduler to find a slot for the aircraft in its schedule and optimize the overall arrival stream schedule based upon the new information. The time needed for scheduling purposes in addition to the theoretical 162 seconds is being called the "coordination time" in Fig. 8.

Operational observations of PDRC at DFW during July 2011 have revealed that the typical departure schedule process is initiated approximately 5 minutes prior to departure during Call For Release situations. According to ATCT and Center personnel this amount of time prior to departure allows for sufficient coordination and meets the minimal need for look-ahead time requirements at DFW. That is not to say that both ATCT and Center don't want the times earlier, but this was an acceptable timeframe for the manually-coordinated tactical departure scheduling process in place today. Considering site feedback and the 2.7 minute flight time difference which would allow these aircraft to compete with non-frozen aircraft in the IAH metered stream, this allows approximately 2.3 minutes of "coordination time" for the tactical departure scheduling process at DFW. It is believed that this look ahead time estimation process can be used for other airports that have a high demand for tactical departure scheduling to identify the look ahead time at which accuracy departure time predictions are needed. Based upon PDRC field test observations as well as data obtained from FAA evaluation of TMA scheduling from air traffic control towers, ${ }^{8,9}$ it is estimated that through automation the "coordination time" taken for the tactical departure scheduling process can be reduced to approximately 30 seconds. Thus, the minimal look ahead time requirement for DFW is $162+30=192$ seconds prior to wheels OFF.

\section{B. Surface prediction accuracy at required look-ahead time for Tactical Departure Scheduling}

The look-ahead time need was based upon relative geometry of the departure airport to the arrival metering freeze horizon plus required coordination time. Look-ahead requirement will likely vary based upon different airport geometry relative to arrival metering freeze horizons, or the airspace geometry associated with EDC flows. Beyond the look-ahead requirement, there remains the question of required departure prediction accuracy at the specified look-ahead time. The departure prediction accuracy requirement may be estimated from observed CFR time compliance in today's tactical scheduling scenario. If surface automation delivers the same level of accuracy provided today by the manual CFR procedure, then it follows that it should provide similar benefit to the existing system. Any increase in the accuracy of the departure prediction times or increased look-ahead time for the prediction would be potentially beneficial to tactical departure scheduling system performance. An additional

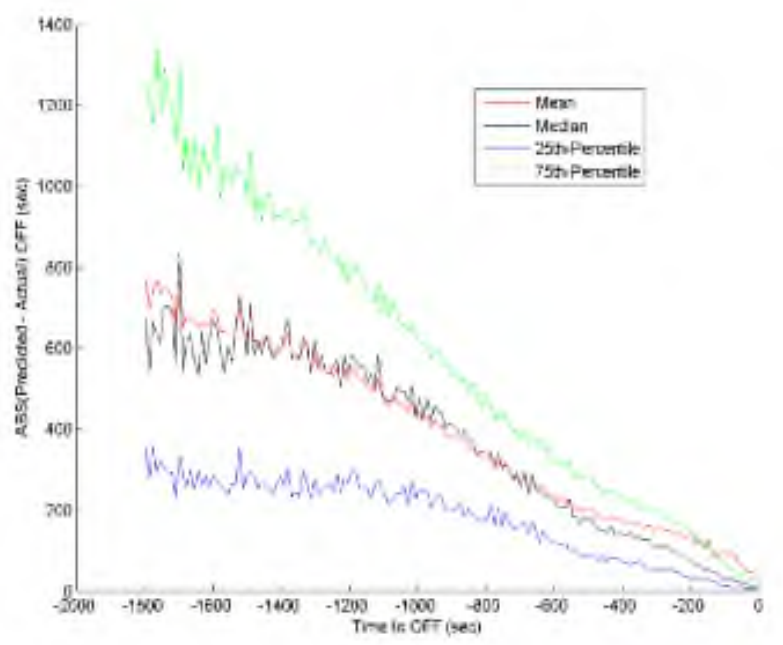

Figure 9. SDSS prediction accuracy at DFW - June 2011. observation to consider is that workload associated with the manual CFR procedure may lead to relatively infrequent use. Any automation that may help reduce the workload threshold at which this level of accuracy could be obtained would likely be used more frequently, which would potentially lead to increased benefits. Another factor to consider is that of any uncertainty that is the result of manual entry or miscommunications like those reports in a companion paper. ${ }^{1}$

Currently, the manual CFR procedure must deliver OFF times that comply with a $-2 /+1$ minute window. Based upon tactical departure scheduling data for the month of January 2011, this time window is being met approximately $62 \%$ of the time by ATCT control of flights to meet their CFR coordinated OFF time. Based upon measurements obtained of the Surface Decision 
Support System (SDSS) accuracy in June of 2011, SDSS can predict aircraft wheels OFF at the same level of controlled CFR flights at approximately 137 seconds prior to OFF time. That is to say that without any CFR manual coordination required (e.g. closed loop system); SDSS can achieve similar levels of predictive accuracy as departure time compliance being achieved today through the CFR process at 137 seconds prior to departure. To meet the tactical departure scheduling requirements for DFW, this level of accuracy must be extended at least to the point of 162 seconds as mentioned previously including any coordination time required for the tactical departure flight. However, it is not necessarily true that SDSS must provide this level of accuracy out to the five minutes which current DFW procedure provides. This is due to the coordination time required when using automation is expected to be reduced from the time it takes in the current procedure. During the initial evaluation of PDRC the focus was on establishing confidence in the surface and en route scheduling components, not on reducing the time period it takes for tactical departure scheduling to occur. Future evaluations should work to increase the amount of look ahead time that accurate OFF time predictions are available while reducing the amount of coordination time required for the tactical departure scheduling process.

Work is currently underway to increase the accuracy of the existing surface management system's predictive capability for those aircraft which have acquired surface surveillance. In addition to the increasing the system's predictive accuracy, areas of research that are recommended are: stability of the OFF time estimates which are provided to the downstream scheduler, utilization of departure prediction confidence in tactical departure scheduling, evaluation of tactical scheduling methods which require OFF time estimates in excess of 10 minutes prior to departure and expansion of OFF time estimates to include airports without ASDE-X surveillance capability.

\section{NAS facilities likely to have greatest benefit from PDRC Technology}

Given knowledge of the current tactical departure scheduling demand at each NAS facility, as well as estimated look ahead time requirements for each facility based upon geometry like that illustrated in Fig. 8, a list of the top NAS facilities which would benefit from PDRC technology was constructed. This survey focused on inbound tactical departure scheduling since $86.5 \%$ of tactical departure scheduling ground delay incurred in the NAS today is scheduled in this manner.

The estimation methodology begins with sites that have a proven demand for tactical departure scheduling like

Table 4. Sites which would benefit from PDRC technology - Jan 2011.

\begin{tabular}{|c|c|c|c|c|c|}
\hline \begin{tabular}{|} 
Scheduled \\
From \\
Airport Code
\end{tabular} & Scheduling From Airport Name & $\mid \begin{array}{c}\text { Scheduled Into } \\
\text { Metered Airport } \\
\text { Code }\end{array}$ & Scheduling Into Metered Airport Name & $\begin{array}{l}\text { Hours } \\
\text { Delay }\end{array}$ & $\begin{array}{c}\text { Number of } \\
\text { Scheduled } \\
\text { Aircraft }\end{array}$ \\
\hline KMCO & Orlando International & KATL & Hartsfield - Jackson Atlanta International & 47.9 & 628 \\
\hline KMEM & Memphis International & KATL & Hartsfield - Jackson Atlanta International & 38.0 & 381 \\
\hline KATL & Hartsfield - Jackson Atlanta International & KCLT & \begin{tabular}{|c|} 
Charlotte/Douglas International \\
\end{tabular} & 32.4 & 426 \\
\hline KBOS & Logan International & KPHL & Philadelphia International & 28.0 & 385 \\
\hline KLAS & Mc Carran International & KLAX & Los Angeles International & 18.8 & 381 \\
\hline KIAD & Washington Dulles International & KCLT & Charlotte/Douglas International & 17.4 & 263 \\
\hline KDTW & Detroit Metropolitan Wayne County & $\mathrm{KPHL}$ & Philadelphia International & 16.1 & 278 \\
\hline KSDF & Louisville International & KATL & Hartsfield - Jackson Atlanta International & 15.9 & 230 \\
\hline KCLE & Cleveland-Hopkins International & $\mathrm{KPHL}$ & \begin{tabular}{|c|} 
Philadelphia International \\
\end{tabular} & 15.7 & 203 \\
\hline KLAX & Los Angeles International & KLAS & Mc Carran International & 15.4 & 318 \\
\hline KSFO & San Francisco International & KLAX & Los Angeles International & 15.0 & 333 \\
\hline KDFW & Dallas/Fort Worth International & $\mathrm{KIAH}$ & George Bush Intercontinental/Houston & 13.3 & 168 \\
\hline KCVG & Cincinnati/Northern Kentucky International & KCLT & Charlotte/Douglas International & 12.9 & 258 \\
\hline KDCA & Ronald Reagan Washington National & KCLT & Charlotte/Douglas International & 12.0 & 246 \\
\hline KBWI & Baltimore/Washington International & KCLT & Charlotte/Douglas International & 11.4 & 271 \\
\hline $\mathrm{KPHX}$ & Phoenix Sky Harbor International & KLAS & Mc Carran International & 11.1 & 196 \\
\hline KCVG & Cincinnati/Northern Kentucky International & KATL & Hartsfield - Jackson Atlanta International & 10.8 & 199 \\
\hline KSAN & \begin{tabular}{|c|} 
San Diego International \\
\end{tabular} & KPHX & \begin{tabular}{|l|} 
Phoenix Sky Harbor International \\
\end{tabular} & 7.3 & 189 \\
\hline $\mathrm{KSJC}$ & Norman Y. Mineta San Jose International & KLAX & Los Angeles International & 7.2 & 168 \\
\hline KLAS & Mc Carran International & $\mathrm{KPHX}$ & Phoenix Sky Harbor International & 6.6 & 200 \\
\hline KMCO & Orlando International & KCLT & Charlotte/Douglas International & 6.1 & 250 \\
\hline KLAX & Los Angeles International & $\mathrm{KPHX}$ & Phoenix Sky Harbor International & 5.7 & 213 \\
\hline KSDF & Louisville International & KCLT & Charlotte/Douglas International & 5.7 & 190 \\
\hline KSNA & John Wayne-Orange County & KLAS & Mc Carran International & 5.5 & 140 \\
\hline KSNA & John Wayne-Orange County & KPHX & Phoenix Sky Harbor International & 3.6 & 173 \\
\hline KSFO & San Francisco International & KLAS & Mc Carran International & 2.6 & 154 \\
\hline
\end{tabular}
those listed in Table 1 . Only the top $10 \%$ users of tactical departure scheduling airport pairs (e.g. KDFW into KIAH) excluding international scheduling were considered. This yielded 81 airports scheduling into 7 different metered airports, each of which tactically scheduled over 130 aircraft during the month of January. The next step was to analyze each departure/arrival airport pair to determine the lookahead time need of each airport, like that illustrated in Fig. 8. In order to include look-ahead time needs that are achievable based upon surface surveillance availability, it was necessary to bound the look-ahead time by the average surface taxi out time. The nationwide average of unimpeded taxi out time of 10.7 minutes was obtained from the FAA's Aviation System Performance Metrics (ASPM) database. Those airports with greater than 10.7 minutes look-ahead time requirement prior to departure were eliminated from the list, which left 55 airports.

The remaining candidate airports were further filtered according to current or planned availability of an ASDE-X surface surveillance system which would allow for trajectory based OFF time estimates to be supplied to the tactical 
departure scheduler. This remaining list consisted of 26 airports, which were ordered by the delay they incurred in January 2011, as listed in Table 4.

The "Scheduling From" column in Table 4 indicates the airport from which tactical departure scheduled aircraft are departing, while the "Scheduled into Metered Airport" indicates the destination of the tactical departure scheduled.

At the top of the list are two airports that are not only ASDE-X equipped, but also have a current Surface Decision Support System (SDSS) adapted. In addition, the third and fourth airports on the list are currently being adapted for the SDSS system in support of other research.

A notable omission from Table 4 is scheduling from Charlotte to Atlanta. While 426 aircraft were tactically scheduled from Charlotte to Atlanta during the month of January, only 35 of these occurred during an Atlanta metering period. The lack of tactical departure scheduling during metering may be due to the 'hybrid metering' design that Atlanta uses in which adjacent centers meters outside of Atlanta Center airspace but the metering advisories are not displayed on Atlanta Center glass.

Analysis of site geometry relative to the freeze horizon indicates that the look-ahead time at which accurate departure predictions are needed becomes greater as the distance from the departure airport within the freeze horizon increases. Inbound tactical departure scheduling analysis has demonstrated that the majority of scheduling occurs near the arrival freeze horizon boundary (11.3 minute average flight time to freeze horizon with 11.4 minute standard deviation). Some of the airports being scheduled from to an arrival metering facility lie geographically inside of the freeze horizon, while others lie outside of the freeze horizon. Heavier usage of tactical departure scheduling near the freeze horizon is consistent with intuition as flights which are sufficiently far away from the TMA freeze horizon generally have sufficient time and space in the arrival stream in order to secure a slot prior to the freeze horizon location. As departing airports get closer to or are within the TMA freeze horizon, the scheduling process becomes more dependent upon the departure prediction accuracy as there is less time for a departing aircraft to compete for resources in the overhead stream while the demand for overhead resources generally also becomes greater. In this manner the geometry of a departure airport relevant to the freeze horizon of the arrival TMA system being scheduled into is an important factor to consider.

Figure 10 illustrates this relationship which is being referred to as the 'Goldilocks Zone' in which achievable levels of departure prediction accuracy can be used for tactical departure scheduling. The following example

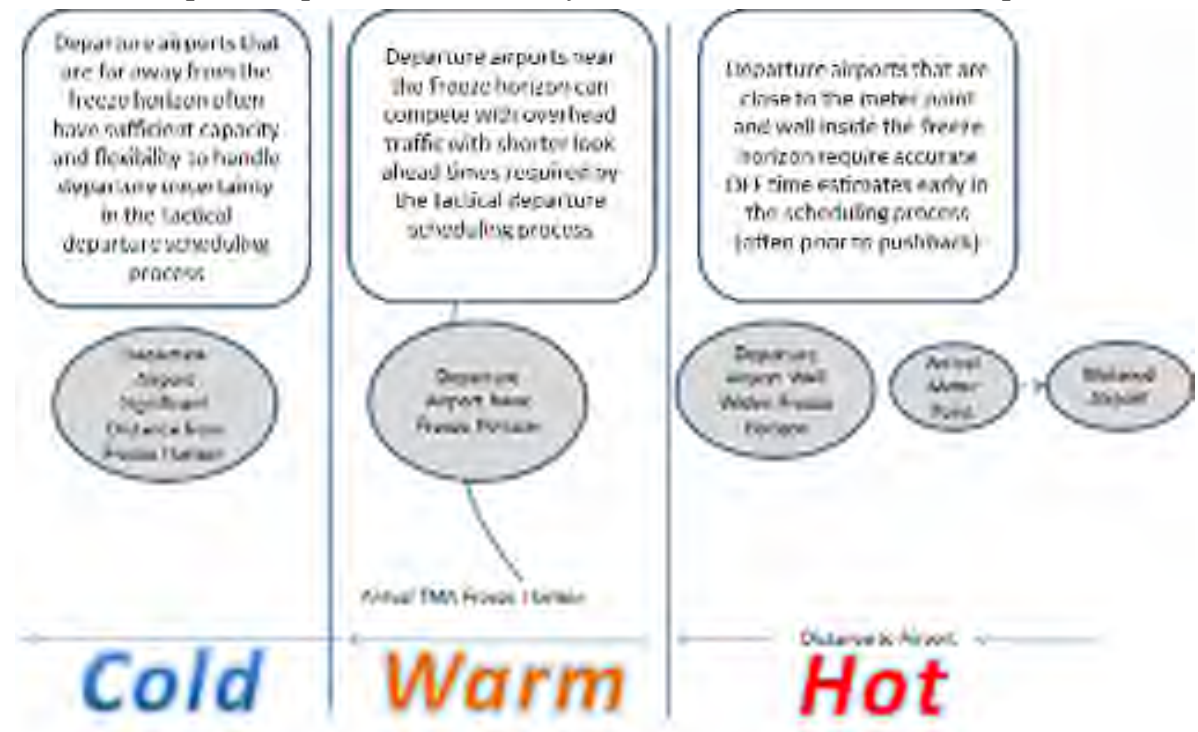

Figure 10. Inbound tactical departure scheduling 'Goldilocks Zone' relationship between departure airport location and freeze horizon. considers if a departure airport requires 15 minutes flying time within the arrival freeze horizon to an arrival metering facility. To actively compete with non-frozen aircraft which are currently airborne in the arrival stream, the look-ahead time predictions must be accurate enough for TMA at least 15 minutes prior to departure. Any error in the departure prediction estimate scheduled at this point will directly impact the arrival stream efficiency as well as controller workload if the sector controller meter list is rippled due to changes. On the other hand if the departure airport is 60

minutes flying time outside of the freeze horizon, then despite the level of departure prediction accuracy, the aircraft will likely have adequate time to be scheduled into the arrival TMA system. 


\section{Conclusions}

Analysis of operational TMA and EDC data from all current deployed facilities covering over 1,082,000 flights during the month of January 2011 indicates that these tactical departure scheduling capabilities are widely used in the NAS today with over 65,000 scheduled aircraft per month using these methods. Increased utilization of tactical departure scheduling decision support tools has been fueled by expansion of adjacent center metering and nation-wide deployment of the EDC capability.

Although tactical departure scheduling with TMA and EDC has become a widely used component in NAS operations today and represents a significant improvement over the previous process which lacked trajectory based ascent modeling, analysis of the current system's performance indicates that significant room for improvement exists by reducing departure time uncertainty. Based upon operational data analysis described in this paper, 6,792 inbound tactically scheduled aircraft and 1,911 outbound tactically scheduled aircraft in January 2011 NAS wide are estimated to have missed the airspace slot they were scheduled into due to departure time prediction uncertainty. The effect to the NAS of a missed scheduled departure slot often leads directly to lost capacity, most notably in the case in which an aircraft is scheduled frozen into an arrival TMA slot but does not meet its expected departure time window. However, measuring the impact to the NAS of a missed departure slot is not always straightforward as some ability to recover the airspace resources exists, often at the cost of additional TMC or controller workload and/or inefficient flight paths.

While the shortfalls of the existing tactical departure scheduling system have become more evident and quantifiable, solutions to these shortfalls are in early stages of maturity relative to other NAS systems. Determining the level of predictive accuracy that trajectory based OFF time predictions must attain for tactical departure scheduling delay reduction benefit is complicated by the lack of surface automation available in operations today and the challenges associated with evaluating a passive OFF time estimation process. This paper proposes metrics and methods to estimate the look ahead time requirement of surface predictions, as well as to identify target airports that are likely candidates for NAS deployment of PDRC technology based upon the departure airport's geometry relative to areas of high airspace demand like those encountered near time based metering freeze horizons. Indications are that departure prediction accuracy requirements for tactical departure scheduling in the NAS are likely not a single value, but rather a range of values that vary in significant part based upon site specific geometry and airspace demand.

\section{References}

${ }^{1}$ Engelland, S., Capps, A, "Trajectory-Based Takeoff Time Predictions Applied to Tactical Departure Scheduling: Concept Description, System Design, and Initial Observations", submitted to AIAA 11 th Aviation Technology, Integration, and Operations (ATIO) Conference, Virginia Beach, VA., September 20-22, 2011.

${ }^{2}$ Palopo,K., Lee, H, Chatterji, G., "Benefit Assessment of the Precision Departure Release Capability Concept", submitted to AIAA 11 th Aviation Technology, Integration, and Operations (ATIO) Conference, Virginia Beach, VA., September 20-22, 2011.

${ }^{3}$ Landry, S. J., and Villanueva, A., AIAA-2007-7713, "Mitigating the Effect of Demand Uncertainty Due to Departures in a National Time-Based Metering System". AIAA's 7th Annual Aviation Technology, Integration, and Operations (ATIO) Technical Forum, Belfast, Northern Ireland, September 18-20, 2007.

${ }^{4}$ Thipphavong, J., and Landry, S. J., "Effects of the uncertainty of departures on multi-center traffic management advisor scheduling.", AIAA-2005-7301, AIAA's 5th Annual Aviation Technology, Integration, and Operations (ATIO) Technical Forum, Arlington, VA., September 26-28, 2005.

${ }^{5}$ Grabbe, S., "Traffic Management Advisor Flow Programs: an Atlanta Case Study", AIAA Guidance, Navigation, and Control Conference, Portland, Oregon, August 08-11, 2011.

${ }^{6}$ Farley, T. C., Landry, S. J., Hoang, T., Nickelson, M., Levin, K. M., Rowe, D., and Welch, J. D., "Multi-Center Traffic Management Advisor: Operational Test Results," AIAA-2005-7300, Proceedings of the 5th AIAA Aviation Technology, Integration, and Operations (ATIO) Conference, Arlington, VA, September 26-28, 2005.

${ }^{7}$ Krozel, J., Rosman, D., Grabbe, S., “Analysis Of En Route Sector Demand Error Sources”, AIAA-2002-5016, AIAA

Guidance, Navigation, and Control Conference and Exhibit, Monterey, California, August 5-8, 2002.

${ }^{8}$ Doble, N., Timmerman, J., Carniol, T., Klopfenstein, M., Tanino, M., and Sud, V., "Linking Traffic Management to the Airport Surface: Departure Flow Management and Beyond," Eighth USA/Europe Air Traffic Management Research and Development Seminar (ATM2009), Napa, CA, 29 Jun - 2 Jul 2009.

${ }^{9}$ Futato, S., McMillan, K., Callon, S., "TMA En Route Departure Capability Jacksonville ARTCC and Orlando ATCT Usage Data", April 8, 2008. 